\title{
Terahertz Emission from Narrow Gap Semiconductors Photoexcited by Femtosecond Laser Pulses
}

\author{
R. Adomavičius*, A. Urbanowicz, G. Molis \\ AND A. KRotKus \\ Semiconductor Physics Institute, A. Goštauto 11, 01108, Vilnius, Lithuania \\ Large increase in the emitted terahertz power was observed for $p$-InAs \\ samples with the $p$-doping levels of approximately $10^{16}-10^{17} \mathrm{~cm}^{-3}$. This \\ increase was explained by a large surface depletion layer and an electric-field- \\ -induced optical rectification effect in the layer. Terahertz fields radiated by \\ the samples of all three investigated $\mathrm{Cd}_{x} \mathrm{Hg}_{1-x}$ Te layers was of the same \\ order of magnitude. No azimuthal angle dependence of the radiated signal \\ was detected, which evidences that linear current surge effect is dominating \\ over nonlinear optical rectification. Azimuthal angle and magnetic fields \\ emission witness that it is caused by linear photo-Dember type processes.
}

PACS numbers: 42.65.Re, 07.57.Hm, 78.47.+p, 72.30.+q

\section{Introduction}

Various terahertz $(\mathrm{THz})$ radiation mechanisms activated by femtosecond laser pulses have been extensively studied recently, including photoconductive switching [1], surface-field emission [2], and optical rectification [3]. Narrow gap semiconductors have attracted much attention after InAs, one of such semiconductors, was found to have the highest emission efficiency of all bulk semiconductors [4]. However, no systematic investigation of $\mathrm{THz}$ radiation in differently doped InAs was performed until now. In the majority of references only nominally undoped or lightly doped (electron or hole density of the order of $10^{16} \mathrm{~cm}^{-3}$ ) InAs crystals were used.

In the present work we measured $\mathrm{THz}$ radiation emitted from laser illuminated surfaces of a large number of variously doped InAs crystals. Another goal of the present work was to investigate $\mathrm{THz}$ radiation from $\mathrm{Cd}_{x} \mathrm{Hg}_{1-x} \mathrm{Te}$ crystals, narrow gap semiconductor compounds that are widely used in infrared devices.

*corresponding author; e-mail: ramunas@opel2.pfi.lt 


\section{Experimental}

THz emission was observed from mechanically polished (111) surfaces. $150 \mathrm{fs}$ duration, $76 \mathrm{MHz}$ repetition rate, and $810 \mathrm{~nm}$ central wavelength pulses from a mode-locked Ti:sapphire laser were used for the sample excitation. $\mathrm{THz}$ radiation was collected by an ultrafast photodetector with dipole antenna and a substrate lens from high resistivity silicon crystal. The detector was manufactured from the layer of GaAs that was grown by molecular beam epitaxy at lower than usual $\left(\approx 250^{\circ} \mathrm{C}\right)$ substrate temperature and had an electron trapping time of $\approx 200 \mathrm{fs}$. Terahertz radiation from $\mathrm{Cd}_{x} \mathrm{Hg}_{1-x} \mathrm{Te}$ and InAs samples excited by femtosecond Ti:sapphire laser pulses was measured. The samples investigated were $n$ - and $p$-type single InAs crystals with the doping levels varying from $10^{16}$ to $10^{19} \mathrm{~cm}^{-3}$, and $\mathrm{Cd}_{x} \mathrm{Hg}_{1-x} \mathrm{Te}$ epitaxial layers with $x=0,0.2$, and 0.3 .

\section{Results and discussion}

Figure 1 shows the dependence of the emitted $\mathrm{THz}$ field amplitude on crystal doping level. It can be seen that there is a strong enhancement of the radiated field for $p$-type doping levels from $10^{16}$ to $10^{17} \mathrm{~cm}^{-3}$. The shape of $\mathrm{THz}$ transients recorded at largest magnitudes was very sensitive to the azimuthal angle of the sample orientation around the axis normal to the surface. For the sample with $p$-doping level in the range of maximal $\mathrm{THz}$ amplitudes, the azimuthal dependence is much pronounced: the ratio of the signals at the angles corresponding to their maxima and minima reaches 20 , whereas for $n$-InAs this ratio is close to the value of 2 , which was observed earlier in [5]. The azimuthal angle dependence measurements are traditionally used for separating linear (photocurrent surge) and nonlinear (optical rectification) contributions to the $\mathrm{THz}$ radiation emission [5]. The measured azimuthal dependences show a clear periodicity of $\cos (3 \theta)$, where

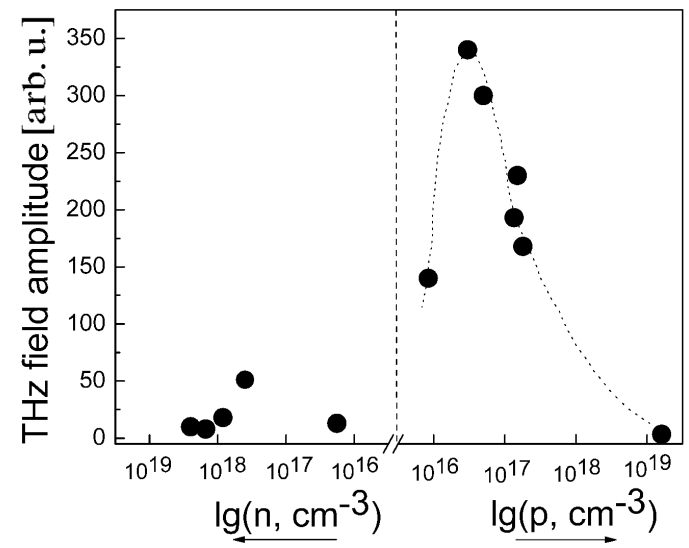

Fig. 1. Dependence of the emitted $\mathrm{THz}$ field magnitude on the InAs crystal doping level (dotted line is guide for eye). 
$\theta$ is the azimuthal angle of the sample orientation around the axis normal to the surface, which corresponds to the optical rectification effect at (111) surfaces. Such a periodicity in the (111) plane is characteristic of both bulk and electric-field-induced optical rectification $[3,6]$. Thus azimuthal angle dependencies alone are not able to discriminate relative influences of these two effects. However, strong doping level dependence in $p$-InAs samples points out that, at least in this case, the prevailing physical mechanism of THz emission is the electric-field-induced instantaneous polarization. Surface depletion layer width in the samples with $p=10^{17} \mathrm{~cm}^{-3}$ is approximately the same as the light absorption length at the laser wavelength $(\approx 100 \mathrm{~nm})$, therefore, a nonlinear optical interaction due to electric-field-induced optical rectification should be most efficient in this case.

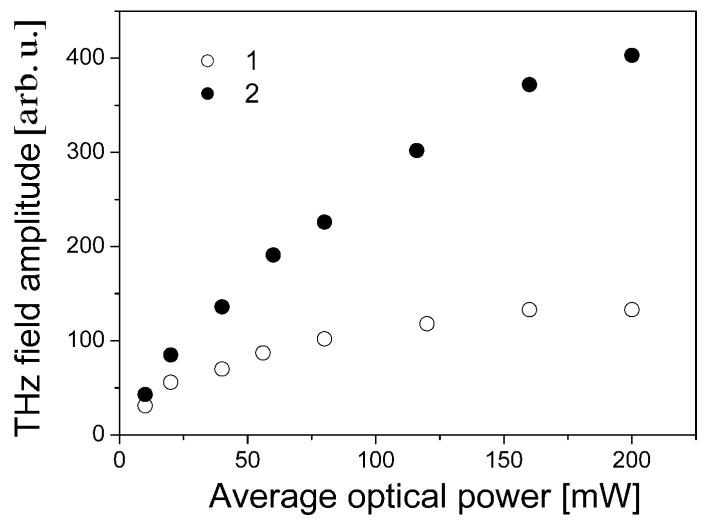

Fig. 2. Optical intensity dependences of the $\mathrm{THz}$ transient magnitudes measured at two azimuthal angles corresponding to the weakest (1) and the strongest (2) THz emission.

Relative role of this nonlinear optical process increases with increasing light intensity. This is illustrated in Fig. 2, where the intensity dependences of the $\mathrm{THz}$ transient magnitude measured at two azimuthal angles corresponding to the strongest and the weakest $\mathrm{THz}$ emission are shown. These dependences clearly show that the $\mathrm{THz}$ emission from $p$-InAs sample is caused by at least two different physical mechanisms. At low optical intensities when angular dependence of the radiated signal is weak, most probably, the current surge due to the transport of photoexcited carriers prevails. With increasing intensity this effect is overpowered by the instantaneous polarization due to the electric-field-induced optical rectification. This assumption is additionally supported by two additional experimental observations. Firstly, the radiated $\mathrm{THz}$ transient magnitude saturates faster for the crystalline orientation corresponding to the minima of the angular dependence than to the maxima. The screening of the surface field that is most probable cause of this saturation would have a stronger influence on the carrier transport than on the nonlinear optical interaction, which will occur on a shorter time scale. Secondly, weak external magnetic field $(\approx 0.28 \mathrm{~T})$ mainly affects the $\mathrm{THz}$ field 
at azimuthal angles where nonlinear optical component is set to a minimum, and where the component due to the photoexcited carrier transport is prevailing.

Figure 3 shows the time-domain wave forms measured on $n$-InAs and three $\mathrm{Cd}_{x} \mathrm{Hg}_{1-x} \mathrm{Te}$ samples with different alloy composition. Peak field values in the majority of $\mathrm{THz}$ transients measured on $\mathrm{Cd}_{x} \mathrm{Hg}_{1-x}$ Te samples are only 2-3 times smaller than in $n$-type InAs, however in the latter case positive peak is followed by a negative dip of nearly the same magnitude leading to much larger enhancement of the emitted $\mathrm{THz}$ power.

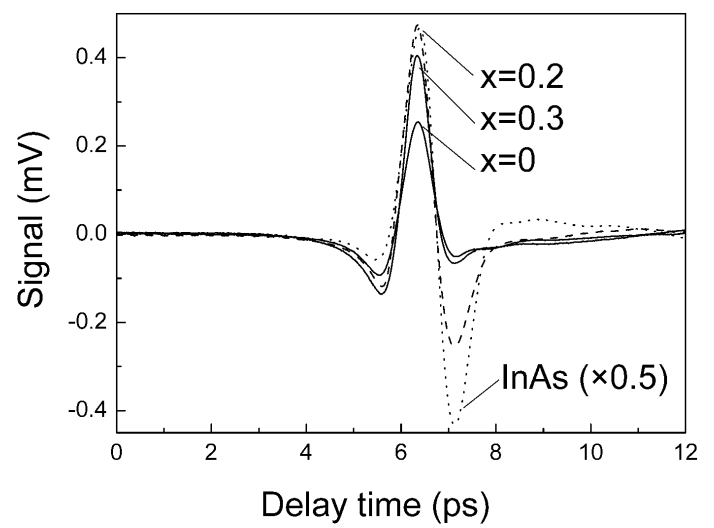

Fig. 3. THz field transients measured for the samples from different narrow-gap semiconductors. The signal was measured by means of a photoconducting antenna and lock-in amplifier system. A part of the laser beam was used to excite the antenna at a variable time delay with respect to $\mathrm{THz}$ pulse.

The magnitude of the $\mathrm{THz}$ field radiated from $\mathrm{Cd}_{x} \mathrm{Hg}_{1-x} \mathrm{Te}$ surfaces does not depend on the azimuthal angle of sample orientation, which can be considered as an evidence of a prevailing role of linear processes in $\mathrm{THz}$ radiation from these materials. The fact that $\mathrm{THz}$ emission from the surfaces of $\mathrm{Cd}_{x} \mathrm{Hg}_{1-x} \mathrm{Te}$ crystals is caused by fast changing photocurrent is also confirmed by the experiment on the magnetic field influence on the shape of the transients. Relatively weak field $(B=0.28 \mathrm{~T})$ of a permanent magnet parallel to the illuminated surface of the sample leads, depending on its polarity, to the increase or the decrease in $\mathrm{THz}$ transient magnitude. This observation is consistent with the model explaining the magnetic field influence by the Lorentz-force-induced rotation of the radiating dipole orientation.

Fast changing photocurrent at semiconductor surface can originate from two physical effects: the acceleration of photoexcited carriers by the built-in field in the surface depletion layer or the photo-Dember effect caused by the different electron and hole diffusion rates. In $\mathrm{Cd}_{x} \mathrm{Hg}_{1-x} \mathrm{Te}$ the surface field will be weak because of a small energy band gap, whereas the carrier diffusion processes will be rather intense due to a short light absorption lengths. Consequently, the ultrafast photocurrent 
transients due to the photo-Dember effect may be the main mechanism of $\mathrm{THz}$ radiation emission from these semiconductors.

\section{Conclusions}

In conclusion, we measured $\mathrm{THz}$ radiation from a set of differently doped InAs samples excited by femtosecond laser pulses. Strong enhancement of the radiation efficiency in $p$-type samples as well its sensitivity to the sample's orientation around the normal to its surface was evidenced. It was also concluded that these experimental observations can be explained by an influence of instantaneous polarization due to electric-field-induced optical rectification at the semiconductor surface.

We observed $\mathrm{THz}$ emission from the surfaces of $\mathrm{Cd}_{x} \mathrm{Hg}_{1-x}$ Te samples excited by femtosecond laser pulses. Azimuthal angle and magnetic fields emission witness that it is caused by linear photo-Dember type processes.

\section{Acknowledgments}

This work was in part supported by NATO "Science for Peace" program (projects SfP-977978 and SfP-974476).

\section{References}

[1] D. Krokel, D. Grischkowsky, M.B. Ketchen, Appl. Phys. Lett. 54, 1046 (1989).

[2] Q. Wu , X.-C. Zhang, Appl. Phys. Lett. 56, 886 (1990).

[3] P.N. Saeta, B.I. Greene, S.L. Chuang, Appl. Phys. Lett. 63, 3482 (1994).

[4] N. Sarukura, H. Ohtake, S. Izumida, Z. Liu, J. Appl. Phys. 84, 654 (1998).

[5] P. Gu, M. Tani, S. Kono, K. Sakai, X.-C. Zhang, J. Appl. Phys. 91, 5533 (2002).

[6] S. L. Chuang, S. Schmitt-Rink, B.I. Greene, P.N. Saeta, A.F.J. Levi, Phys. Rev. Lett. 68, 102 (1992). 